based on the data from appendix $\mathrm{C}$, but in fact it ignores all the data collected since 1956. The figure showing the British distribution of melanics, page 135 , has also been lifted bodily from the same paper and has not been updated, thus it loses a great deal of its value.

Despite these serious criticisms, and many more, the volume is clearly essential reading for students of microevolution. One of its very great merits is the large number of speculative conclusions which have never been published before, and which will be a fruitful source of future experiments for many years to come. If the author had taken a great deal more trouble with the book and the publishers had made sure the plates were inserted in the right places (they have not in my copy), this book, instead of being a very interesting and useful volume, would have been a brilliant one.

P. M. Sheppard

\section{Estimating Animals}

The Estimation of Animal Abundance and Related Parameters. By G. A. F. Seber. Pp. xii +56. (Griffin: London, May 1973.) $£ 12$.

Often a first problem in ecology is to devise a census technique. Rarely are direct absolute counts possible and recourse must be made to sampling methods. Many of these have been derived by statisticians and mathematicians and they answer the purpose just so long as the simplifying assumptions on which they are based are applicable. This book, written by an authority in statistics (indeed the author is Professor of Statistics at Auckland University), brings together the various mathematical techniques for estimating animal numbers and such related parameters as birth, mortality and migration rates. It is certainly useful to have all these techniques presented as a reference text which adopts a standardised notation, defined in a preliminary chapter, but with this somewhat esoteric content the book is rather expensive. Its value to a practising ecologist must depend on its accuracy, and its completeness, and perhaps also on any critical discussion of the validity and scope of the techniques mentioned.

On the first point the book certainly scores, for the field has been surveyed comprehensively and well. Each major topic, for example, the chapter dealing with "mark releases before the sampling period in open populations", is broken down to such categories as "single release: instantaneous sampling" or "single release and continuous sampling: mortality estimates" and under this last head maximum-likelihood and regression estimates are considered. In this way, and throughout the book, the final classification usually resolves into a particular mathematical technique defined by equations and followed by one or two worked examples from the literature.

Printers and bad proof reading can make 'hash and blotty' out of mathematical equations and symbols-indeed, some of the original published methods have appeared with irritating errors such as wrong signs. At a quick check I have found no errors in the formulae but my search has not been exhaustive.

On the third point, the book is a bit disappointing, for it offers no real discussion of the validity of the underlying biological assumptions. In capture-recapture studies it is invariably a necessary assumption that every animal in the population, whether marked or unmarked, has the same probability of being caught in the $i$-th sample, assuming it to be alive. Real animals rarely satisfy such premises. In fact, it might be suggested that the essence of survival has been the evolution of predictive mechanisms, of information exchange through communal behaviour, of social hierarchies which restrict movement, indeed of a host of conventions which serve to remove any element of chance and therefore confound our simple premises that individuals can be assumed to behave randomly. Perhaps we need some new approaches. In the meanwhile, this book will serve as a helpful reference work for numerate $\begin{array}{ll}\text { experts. } & \text { R. K. MURTON }\end{array}$

\section{Statistics in Medicine}

Causal Thinking in the Health Sciences: Concepts and Strategies of Epidemiology. By Mervyn Susser. Pp. xviii +181 . (Oxford University: London and New York, August 1973.) £2.20.

ONLY the first part of the title of this book appears on the cover, on the fly. leaf, and on each page. This gives a most misleading impression. It leads one to expect a proposal of some novel mode of reasoning, or perhaps a polemic on the inadequacy of current modes of reasoning, about medical practice. Indeed, I missed the title page and read with initial prejudice and with growing surprise. For in fact the book is a systematic examination of epidemiological methods in medicine, that is, of using statistical data to establish the existence of hazards to health and the means of preventing them. This is the discipline that has to its credit the discovery of the means of eliminating cholera before the cholera vibrio was known; the discovery that most bronchial cancer, and most chronic respiratory disease, is linked in some as yet unknown way to the smoking of cigarettes and can be avoided by avoiding such smoking; the discovery that asbestos is capable of causing, by as yet unknown means, cancers of the mesothelial layers of the lung and other viscera.

Epidemiology has also discovered, however, associations between the softness of domestic water supplies and mortality from ischaemic heart disease, and between potato blight and births of children with spina bifida. Whether these associations reflect causal links is still a matter for dispute, and conflicting evidence has been produced. Professor Susser's concern is with the nature of epidemiological evidence, its classification into types, and the discovery of means by which direct causal links can be distinguished from indirect ones and from associations with no causal element at all.

The first six chapters provide an introduction to epidemiology and to the complex interplay between the environment and the population inhabiting it. The discussion is general, though cogent examples are given, often in the form of what the author calls "exhibits"tabular or graphical data displayed as if on a wall-board. The development is clear, but heavy going and handicapped by technical terms of low mnemonic value which are introduced as new factors are recognised to enter the epidemiological situation. One finds, and can in part translate with the aid of the index, sentences such as "The characteristic that distinguishes the component variable from other control variables is its integration, by definition or implication, with the global independent variable under study". Mercifully they are uncommon.

In the remaining five chapters the strategies available to the epidemiologist to elucidate complex associations are examined systematically. These chapters are stimulating and thought provoking, and they succeed to a great extent in reducing to order the variety of difficulties that beset this process. No general principles emerge for recognising the disturbing factors that may be present in a particular case, and the student must learn by analogy to try to guess what they may be by noting what in other cases they have been. Once again, however, the naming of these factors is unhelpful: "When a moderator variable is introduced into an analysis, and reveals interaction, the process is called specification". Incidentally, interaction is here defined as the difference between the mean effects of one factor when another is present or absent. This is twice the size of the interaction according to the conventional statistical definition.

The book can be strongly recommended, not only to epidemiologists, but also to those interested in the findings of epidemiology It represents a major advance in the process of establishing 